TRANSACTIONS OF THE

AMERICAN MATHEMATICAL SOCIETY

Volume 349, Number 6, June 1997, Pages 2253-2269

S 0002-9947(97)01933-8

\title{
SECOND VARIATION OF SUPERMINIMAL SURFACES INTO SELF-DUAL EINSTEIN FOUR-MANIFOLDS
}

\author{
SEBASTIÁN MONTIEL AND FRANCISCO URBANO
}

\begin{abstract}
The index of a compact orientable superminimal surface of a selfdual Einstein four-manifold $M$ with positive scalar curvature is computed in terms of its genus and area. Also a lower bound of its nullity is obtained. Applications to the cases $M=\mathbb{S}^{4}$ and $M=\mathbb{C P}^{2}$ are given, characterizing the standard Veronese immersions and their twistor deformations as those with lowest index.
\end{abstract}

\section{INTRODUCTION}

From a geometrical point of view it is not necessary to justify the study of the second variation operator of minimal surfaces into Riemannian manifolds. In fact, this Jacobi operator carries the information about the stability properties of the surface when it is thought of as a stationary point for the area potential. The first step in this direction was given by Simons [Si], where he characterized the minimal submanifolds of the sphere with the lowest index (number of independent infinitesimal deformations which do decrease the area). Also, the existence and properties of stable minimal surfaces in Riemannian manifolds have been successful used in the study of their topology (see [MM, SY, SiuY]), and, from there, a lot of works have been dedicated to studying stability and index of several types of minimal surfaces in very different ambient spaces. The simplest case is that of codimension one, that is, the case of hypersurfaces where people characterize normally those of lowest index (see [Si, FCS, FC, SY, MoR, U] and references therein). Our knowledge is really small when the codimension is greater than one, and only a few particular situations had been considered [MM, SiuY, E, O]. Very recently Micallef and Wolfson in [MW] have dealt with the codimension two case, giving lower bounds for the index of minimal surfaces in four-dimensional Riemannian manifolds whose curvature satisfies a certain condition which includes important particular cases.

The present work was done before the authors knew of the Micallef and Wolfson paper. In spite of it, our article could be thought of as a continuation of [MW]. In fact, we will deal with a special family of minimal immersions from surfaces into four-manifolds and, although we will consider more restrictive assumptions on our ambient spaces, the most significant examples (from geometrical and physical points of view) will be included among them, as we will point out later.

Received by the editors October 10, 1994 and, in revised form, September 15, 1995.

1991 Mathematics Subject Classification. Primary 53A10; Secondary 49Q20.

Key words and phrases. Superminimal surfaces, index, nullity.

Both authors partially supported by DGICYT grant PB94-0796.

(C)1997 American Mathematical Society 
On the other hand, (branched) minimal immersions from surfaces can be also viewed as conformal harmonic maps, that is, they are stationary points for the energy functional, although stability or index as a minimal surface is not the same as stability or index as a harmonic map. However, in the case where the surface has genus zero the space of (branched) minimal immersions and the space of harmonic maps to any Riemannian manifold coincide. So, in this case, the nullity of the minimal immersion, that is, the dimension of the space consisting of the Jacobi fields (infinitesimal deformations through minimal immersions), informs us about the dimension of the moduli space of harmonic maps to that manifold, up to reparametrizations of the surface.

These considerations make attractive to the authors the study of minimal immersions from compact surfaces into self-dual Einstein four-manifolds. The feature of the dimension four can be strongly used in this situation. In fact, we know, since the beautiful work of Atiyah, Hitchin and Singer [AHS], that the twistor bundle of such a manifold is a complex manifold. So, one can ask for those minimal immersions whose twistor liftings are holomorphic (see $[\mathrm{Fr}]$ ). These are the so-called superminimal immersions, which we will deal with in this paper and which can be considered, in some sense, as an analogue to the self-dual connections. In fact, in this case the second order harmonic equation reduces to a first order one. So, we will study here the Jacobi operator of superminimal immersions from compact orientable surfaces into self-dual Einstein four-manifolds. This includes superminimal immersions into real space forms, complex space forms and self-dual gravitons (for example, all the K3-surfaces with the Calabi-Yau metric). Using the conformal geometry of the surface, we will see that all the eigenspaces of the Jacobi second variation operator of our superminimal immersions are complex in a certain sense and, so, they have even dimensions. We will compute and estimate the multiplicities of its negative and zero eigenvalues. In this way, in Theorem 3 we will show that

the first eigenvalue of the Jacobi operator of a superminimal immersion from a compact orientable surface into a self-dual Einstein four-manifold with scalar curvature $\tau$ is greater than or equal to $-\tau / 6$, and in almost all the cases is exactly that value

and estimate the corresponding multiplicity. In the case where the scalar curvature of the ambient space is positive, we will associate the eigensections of the Jacobi operator corresponding to the non-positive eigenvalues with holomorphic sections of certain holomorphic line bundles on the surface. This will allow us to show in Theorem 4 that

the dimension of the moduli space of superminimal immersions with Euler class $c(N)$ for its normal bundle from a compact orientable surface with genus $g$ into a self-dual Einstein four-manifold with positive scalar curvature is $6(1-g)+2 c(N)$, provided that $c(N)>4(g-1)$, and to compute exactly its index.

Finally, in the last section, we will specialize our methods to the case where the ambient space is the four-sphere or the complex projective plane. A detailed knowledge of the twistor spaces of these two four-manifolds leads us to compute in Corollaries 7 and 8 the dimension of the moduli space of conformal superminimal immersions, 
with a given Euler class (area) for the normal bundle, from a generic compact Riemann surface with small genus ( $g \leq 15$ in the case of the sphere and $g \leq 8$ in the case of the projective plane). Also, in Corollaries 9 and 11

we will be able to characterize the Veronese surfaces and their twistor deformations both in $\mathbb{S}^{4}$ and $\mathbb{C P}^{2}$ as those full superminimal surfaces with lowest index.

In fact, we will see that all the infinitesimal deformations of these surfaces decreasing their areas came from conformal transformations of the ambient spaces. We will finish our work by computing the complete spectrum of the Jacobi operator for these two Veronese surfaces.

The authors are thankful for the valuable suggestions and comments made by the referee.

\section{Superminimal SURfaces}

Let $M$ be a four-dimensional oriented manifold endowed with a Riemannian metric $\langle$,$\rangle . Given a point x \in M$, let $P_{x}$ be the set of almost Hermitian structures $J_{x}$ over $T_{x} M$ such that, if $\Omega(u, v)=\left\langle J_{x} u, v\right\rangle$, then $-\Omega \wedge \Omega$ is the orientation induced on $T_{x} M$ from $M$. Then $P=\bigcup_{x \in M} P_{x}$ is a $\mathbb{C P}^{1}$-fiber bundle over $M$, called the twistor bundle of $M$. If $\pi: P \rightarrow M$ is the projection, then the vertical distribution $V=\operatorname{ker} \pi_{*}$ inherits from the standard complex structure of the complex projective line $\mathbb{C} P^{1}$ an almost complex structure $J^{v}$. The Levi-Civita connection of $M$ induces a decomposition of the tangent bundle of $P$

$$
T P=H \oplus V
$$

with $H \equiv \pi^{*} T M$, and there is also on the horizontal distribution $H$ an almost complex structure $J^{h}$ defined by

$$
J_{J_{x}}^{h}=J_{x} .
$$

So, $\mathbb{J}=J^{h}+J^{v}$ defines an almost complex structure on $P$ which only depends on the oriented conformal structure of $M$. A central result due to Atiyah, Hitchin and Singer $[\mathrm{AHS}]$ is that $(P, \mathbb{J})$ is a complex manifold if and only if the Riemannian metric of $M$ is self-dual.

Consider now an immersion $\phi: \Sigma \rightarrow M$ from an oriented surface $\Sigma$ into an oriented Riemmannian four-manifold $M$. We denote by $J$ the complex structure on $\Sigma$ compatible with the given orientation. If $T^{\perp} \Sigma$ is the normal bundle of $\phi$, then we have the orthogonal decomposition

$$
\phi^{*} T M=T \Sigma \oplus T^{\perp} \Sigma .
$$

Let $\bar{\nabla}$ be the connection on $\phi^{*} T M$ induced from the Levi-Civita connection of $T M$ and let $\bar{\nabla}=\nabla+\nabla^{\perp}$ be the corresponding decomposition.

If $\left\{e_{1}, e_{2}, e_{3}, e_{4}\right\}$ is an oriented orthonormal local reference on $\phi^{*} T M$ such that $\left\{e_{1}, e_{2}\right\}$ is an oriented reference on $T \Sigma$, then we define an almost complex structure, also denoted by $J$, on $T^{\perp} \Sigma$ by

$$
J e_{3}=-e_{4} .
$$

It is easy to check that $\nabla^{\perp} J=0$, and so the Koszul-Malgrange theorem [KM] gives a unique holomorphic structure on $T^{\perp} \Sigma$ such that a section $\xi \in \Gamma\left(T^{\perp} \Sigma\right.$ ) (in 
general, $\Gamma$ will stand for the space of sections of a vector bundle) is holomorphic if and only if $D_{v} \xi=0$ for any $v \in T \Sigma$, where

$$
D_{v} \xi=\nabla \frac{\perp}{J} \xi-J \nabla_{v}^{\perp} \xi \text {. }
$$

We will represent by $N$ this holomorphic line bundle over the surface $\Sigma$.

Also, the almost complex structure, once more denoted by $J$, on $\phi^{*} T M$ defined as the sum of the two above gives to $\phi^{*} T M$ a structure of complex vector bundle over $\Sigma$. In this way we have associated to the immersion $\phi$ an almost Hermitian structure $J_{\phi(p)}$ on $T_{\phi(p)} M$ such that $J_{\phi(p)} \in P_{\phi(p)}$ for each point $p \in \Sigma$. So, one can define the twistor lifting $\tilde{\phi}: \Sigma \rightarrow P$ by

$$
\tilde{\phi}(p)=J_{\phi(p)} \text {. }
$$

Notice that, following this definition, we can dispose of a twistor lifting only for immersions from an oriented surface into an oriented four-manifold. In fact, if we had chosen on $\Sigma$ the opposite orientation, then the new twistor lifting would have been $\tilde{\phi}(p)=-J_{\phi(p)}$.

The immersion $\phi$ is called superminimal if the map $\tilde{\phi}$ is horizontal, that is, $\tilde{\phi}_{*}(T \Sigma) \subset H$. Although it is not explicitly stated, the following result was proved in $[\mathrm{Fr}]$.

Proposition 1. Let $\phi: \Sigma \rightarrow M$ be an immersion from an oriented surface $\Sigma$ into an oriented four-dimensional Riemannian manifold. Then the following assertions are equivalent:

a) $\phi$ is superminimal.

b) $\phi$ is a minimal immersion and the twistor lifting map $\tilde{\phi}:(\Sigma, J) \rightarrow(P, \mathbb{J})$ of $\phi$ is holomorphic.

c) The second fundamental form $\sigma$ of $\phi$ satisfies

$$
\sigma(J u, v)=J \sigma(u, v)
$$

for any vectors $u, v \in T \Sigma$.

d) The almost Hermitian structures field $J$ on $\phi^{*} T M$ is parallel with respect to the induced connection $\bar{\nabla}$, that is, $\bar{\nabla} J=0$.

A consequence of this proposition is that the superminimality of $\phi$ does not depend on the chosen orientation on the surface $\Sigma$, because the two almost complex structures on $\phi^{*} T M$ obtained from the two possible orientations are opposite, and so they are parallel or not simultaneously. Hence, we may talk about superminimal immersions from an orientable surface into an oriented four-dimensional Riemannian manifold.

In this paper we will only consider self-dual Einstein four-manifolds as ambient spaces for our superminimal immersions. This family of Einstein four-manifolds has been extensively studied from different points of view [Be1, Be2] and includes, for instance, orientable real space forms endowed with either of the two possible orientations, complex space forms with the orientation induced from their complex structure, and Ricci-flat Kähler surfaces with the orientation opposite to that induced from their complex structures, for example, K3-surfaces (with any Kähler metric provided from the positive solution to the Calabi conjecture). If the scalar curvature is positive, Hitchin and Friedrich and Grünewald [H, FrG] proved that a compact manifold of this type is isometric either to the sphere $\mathbb{S}^{4}$ with the standard metric or to the complex projective plane $\mathbb{C P}^{2}$ endowed with the Fubini-Study 
metric. If the scalar curvature is zero, Hitchin $[\mathrm{H}]$ proved that a compact self-dual manifold is either flat or its universal covering is a K3-surface with the Calabi-Yau metric.

The space of superminimal immersions from orientable surfaces into the above ambient spaces has been also considered by many authors [Br, CheW, ES, G], and it is big enough. For instance, any compact Riemann surface can be conformally immersed into $\mathbb{S}^{4}$ or $\mathbb{C P}^{2}$ as a superminimal surface. Also, all complex and totally real surfaces into Ricci-flat Kähler surfaces are superminimal.

Let $\phi: \Sigma \rightarrow M$ be our superminimal immersion from the orientable surface $\Sigma$ into the self-dual Einstein four manifold $M$ whose scalar curvature will be represented by $\tau$. The normal curvature $K^{\perp}$ of the immersion $\phi$ is given by

$$
K^{\perp}=R^{\perp}\left(e_{1}, e_{2}, e_{3}, e_{4}\right),
$$

where $R^{\perp}$ is the curvature tensor of the normal connection $\nabla^{\perp},\left\{e_{1}, e_{2}, e_{3}, e_{4}\right\}$ is an oriented local orthonormal reference on $\phi^{*} T M$ and $\left\{e_{1}, e_{2}\right\}$ is a reference on $T \Sigma$. Using the Ricci equation and Proposition 1, we obtain that

$$
K^{\perp}=\bar{R}\left(e_{1}, e_{2}, e_{3}, e_{4}\right)+\frac{|\sigma|^{2}}{2},
$$

where $\bar{R}$ is the curvature tensor of the connection $\bar{\nabla}$. Taking into account that $M$ is self-dual and Einstein, we can prove that

$$
\bar{R}\left(e_{1}, e_{2}, e_{3}, e_{4}\right)+\bar{R}\left(e_{1}, e_{2}, e_{2}, e_{1}\right)=\frac{\tau}{12},
$$

and so the Gauss equation for the immersion $\phi$ tells us that

$$
K+K^{\perp}=\frac{\tau}{12},
$$

where $K$ is the Gauss curvature of the induced metric on $\Sigma$. In the case where $\Sigma$ is a compact surface of genus $g$, if we denote by $A$ the area of the induced metric, integrating (2) over $\Sigma$ we have

$$
\frac{\tau A}{24 \pi}=2(1-g)+c(N)
$$

where $c(N)$ is the Euler characteristic of the line bundle $N$. By the way we remark that $\tau A / 24 \pi$ is an integer which is twice the so-called (in the twistor terminology) twistor degree of $\phi[\mathrm{ES}]$.

\section{Second variation of the ARea}

As we have seen in Proposition 1, all superminimal immersions are minimal, that is, they are critical points for the area functional. So, it is natural to consider the second variation operator for such an immersion $\phi$. This operator, known as the Jacobi operator of $\phi$ and which we will denote by $L$, is a strongly elliptic operator acting on the compactly supported sections of the normal bundle $T^{\perp} \Sigma$. Suppose that $\Sigma$ is compact. Then the Jacobi operator is an endomorphism of the space $\Gamma\left(T^{\perp} \Sigma\right)$ given by $[\mathrm{L}]$ :

$$
L=\Delta^{\perp}+\mathcal{B}+\mathcal{R},
$$

where $\Delta^{\perp}$ is the second order operator

$$
\Delta^{\perp}=\sum_{i=1}^{2}\left\{\nabla_{e_{i}}^{\perp} \nabla_{e_{i}}^{\perp}-\nabla_{\nabla_{e_{i}} e_{i}}^{\perp}\right\}
$$


and $\mathcal{B}, \mathcal{R}$ are defined as follows

$$
\mathcal{B}(\xi)=\sum_{i, j=1}^{2}\left\langle\sigma\left(e_{i}, e_{j}\right), \xi\right\rangle \sigma\left(e_{i}, e_{j}\right), \quad \mathcal{R}(\xi)=\sum_{i=1}^{2}\left(\bar{R}\left(\xi, e_{i}\right) e_{i}\right)^{\perp},
$$

where $\xi \in \Gamma\left(T^{\perp} \Sigma\right),\left\{e_{1}, e_{2}\right\}$ is an orthonormal reference tangent to $\Sigma$ and $\perp$ denotes the normal part of a vector tangent to $M$. Let $Q$ be the quadratic form associated to the Jacobi operator, defined by

$$
Q(\xi)=-\int_{\Sigma}\langle L \xi, \xi\rangle d A .
$$

We will represent by Ind $\phi$ and $\mathrm{Nul} \phi$ the index and nullity of the quadratic form $Q$, which are respectively the number of negative eigenvalues of $L$ and the multiplicity of zero as an eigenvalue of $L$. The eigenspace corresponding to the zero eigenvalue will be refered to as the nullity space of $\phi$ and denoted by $N(\phi)$.

As $\phi$ is superminimal, by using Proposition 1, one checks easily that

$$
\mathcal{B}(\xi)=\frac{|\sigma|^{2}}{2} \xi
$$

Also, we have

$$
\mathcal{R}(\xi)=\sum_{\alpha=3}^{4} \sum_{i=1}^{2} \bar{R}\left(\xi, e_{i}, e_{i}, e_{\alpha}\right) e_{\alpha}=\frac{\tau}{4} \xi-\bar{R}_{3443} \xi .
$$

But, as $M$ is self-dual and Einstein, $\bar{R}_{3443}=\bar{R}_{1221}$. Now, from the Gauss equation, we obtain that

$$
\mathcal{R}(\xi)=\frac{\tau}{4} \xi-K \xi-\frac{|\sigma|^{2}}{2} \xi,
$$

and so the Jacobi operator $L$ is given by

$$
L \xi=\Delta^{\perp} \xi-K \xi+\frac{\tau}{4} \xi .
$$

A direct consequence of this expression obtained for the Jacobi operator of a superminimal immersion is the following result.

Proposition 2. The Jacobi operator $L$ of any superminimal oriented surface of a self-dual Einstein four-manifold is J-invariant, that is, it commutes with the almost Hermitian structure $J$ defined on the normal bundle. In particular, the multiplicity of all its eigenvalues and, so, its index and its nullity are even.

We continue by proving the following result, which is a slight extension (see the first Remark after its proof) of Theorem 5.10 in [MW]. We include it for the sake of completeness.

Theorem 3. Let $\phi: \Sigma \rightarrow M$ be a superminimal immersion from a compact orientable surface $\Sigma$ of genus $g$ into a self-dual Einstein four-dimensional manifold $M$ with scalar curvature $\tau$, and let $\lambda_{1}$ be the first eigenvalue of the Jacobi operator of $\phi$. Then $\lambda_{1} \geq-\tau / 6$. Moreover

a) Suppose that $\tau=0$. If $g \geq 2$, then $\lambda_{1}=0$ with multiplicity $2 g$ or $2 g-2$. If $g=1$, then $\lambda_{1}=0$ with multiplicity 2 or $\lambda_{1}>0$, according as the normal bundle $N$ is trivial or not. If $g=0$, then $\lambda_{1}>0$.

b) If $\tau>0$, then either $g=0$ and $\phi$ is a stable immersion with $A=\frac{24 \pi}{\tau}$, or $\lambda_{1}=-\frac{\tau}{6}$ with multiplicity $\frac{\tau A}{12 \pi}+2 g-2$, where $A$ is the area of the metric on $\Sigma$ induced from $\phi$. 
Proof. From (4) and (5) and integrating by parts we obtain that

$$
Q(\xi)=\int_{\Sigma}\left\{\left|\nabla^{\perp} \xi\right|^{2}+K|\xi|^{2}-\frac{\tau}{4}|\xi|^{2}\right\} d A
$$

On the other hand, from (1) we obtain

$$
\frac{1}{2}|D \xi|^{2}=\left|\nabla^{\perp} \xi\right|^{2}-\sum_{i=1}^{2}\left\langle\nabla_{J e_{i}}^{\perp} \xi, \nabla_{e_{i}}^{\perp} J \xi\right\rangle
$$

where $\left\{e_{1}, e_{2}\right\}$ is an orthonormal local reference on $\Sigma$. Now we are going to compute the integral of the sum above. Integrating by parts, we have that

$$
-\int_{\Sigma} \sum_{i=1}^{2}\left\langle\nabla_{J e_{i}}^{\perp} \xi, \nabla_{e_{i}}^{\perp} J \xi\right\rangle d A=\int_{\Sigma} \sum_{i=1}^{2}\left\langle\nabla_{e_{i}}^{\perp} \nabla_{J e_{i}}^{\perp} \xi, J \xi\right\rangle d A .
$$

But

$$
\sum_{i=1}^{2} \nabla_{e_{i}}^{\perp} \nabla_{J}^{\perp} e_{i} \xi=\frac{1}{2} \sum_{i=1}^{2} R^{\perp}\left(e_{i}, J e_{i}\right) \xi=-K^{\perp} J \xi .
$$

The two expressions above together with (2) and (6) give

$$
Q(\xi)=\int_{\Sigma}\left\{\frac{1}{2}|D \xi|^{2}-\frac{\tau}{6}|\xi|^{2}\right\} d A
$$

This expression for the quadratic form $Q$ has been already obtained by Micallef and Wolfson (see $[\mathrm{MW}]$, p. 264). Hence, it is clear that the first eigenvalue of $L$ is greater than or equal to $-\frac{\tau}{6}$, and it is exactly this quantity if and only if $\operatorname{dim} H^{0}(\Sigma, \mathcal{O}(N))$ is positive, where, in general we will use $H^{q}(\Sigma, \mathcal{O})$ for the $q$-th cohomology group of $\Sigma$ with coefficients in the sheaf of holomorphic sections of some line bundle on $\Sigma$. Now the Riemann-Roch theorem, jointly with the duality of Serre and formula (3), which computes the Euler characteristic for the normal holomorphic line bundle $N$, tell us that

$$
\begin{aligned}
\operatorname{dim} H^{0}(\Sigma, \mathcal{O}(N)) & =1-g+c(N)+\operatorname{dim} H^{1}(\Sigma, \mathcal{O}(N)) \\
& =g-1+\frac{\tau A}{24 \pi}+\operatorname{dim} H^{0}\left(\Sigma, \mathcal{O}\left(\kappa \otimes N^{*}\right)\right),
\end{aligned}
$$

where $N^{*}$ means the dual or conjugate line bundle of $N$ and $\kappa$ is the canonical line bundle over $\Sigma$. But, we have

$$
c\left(\kappa \otimes N^{*}\right)=c(\kappa)-c(N)=-\frac{\tau A}{24 \pi} .
$$

So, if $\tau=0$, then $\operatorname{dim} H^{0}\left(\Sigma, \mathcal{O}\left(\kappa \otimes N^{*}\right)\right)$ is either one or zero according to whether the line bundle $\kappa \otimes N^{*}$ is trivial or not. Using (7), our assertion in a) is proved.

If $\tau$ is positive, then $\kappa \otimes N^{*}$ is a negative line bundle and so it has no global sections on $\Sigma$. So, in this case, formula (7) gives us

$$
\operatorname{dim} H^{0}(\Sigma, \mathcal{O}(N))=\frac{\tau A}{24 \pi}+g-1
$$

From here, the only case where $\operatorname{dim} H^{0}(\Sigma, \mathcal{O}(N))$ is zero corresponds to $g=0$ and $A=\frac{24 \pi}{\tau}$. In this case, Theorem 4 below will tell us that the immersion is stable.

Remark. As a consequence of Theorem 3 we obtain that the immersion is stable when the scalar curvature $\tau$ is non-positive, and its nullity is zero when $\tau$ is negative. This result has been proved in [MW], Theorem 5.10. 
Remark. If the ambient space $M$ is the sphere $\mathbb{S}^{4}$, it is already known [Si] that $-\frac{\tau}{6}$ (which is -2 when the curvature of the four-sphere has been taken to be one) is an eigenvalue for the Jacobi operator $L$. So, in this case, there are no stable two-spheres. If $M$ is the complex projective plane $\mathbb{C P}^{2}$, then $g=0$ and $\tau A=24 \pi$ only happens (see [EGT]) when $\phi$ is an embedding of a Riemann two-sphere over a complex projective line of $\mathbb{C P}^{2}$.

Now, we will state and prove our main result in this paper, where we will be able to compute exactly the index of any orientable compact superminimal surface into a self-dual Einstein four-manifold with positive scalar curvature and give strong estimates for its nullity. It is not usual to calculate precisely the index and the nullity for minimal submanifolds, but only to give some estimates which are attained at isolated examples. The method of the proof is to observe that the operator $L-\frac{\tau}{6}$ on the normal bundle looks like a Laplacian operator and to obtain an integral formula by making standard manipulations of Weitzenböck formulae type. In a certain sense, our idea could recall the estimate for the first eigenvalue of the Laplacian for Riemannian manifolds whose Ricci curvature is bounded below by a positive constant given by Lichnerowicz and Obata (see, for instance, [Ch], p. 82).

Theorem 4. Let $\phi: \Sigma \rightarrow M$ be a superminimal immersion from an orientable compact surface $\Sigma$ of genus $g$ into a self-dual Einstein four-dimensional manifold with positive scalar curvature $\tau$. Denote by $A$ the area of the immersion. Then

$$
\mathrm{Nul} \phi \geq \frac{\tau A}{12 \pi}+2-2 g \quad \text { and } \quad \text { Ind } \phi=\frac{\tau A}{12 \pi}+2 g-2 .
$$

Moreover, if $\tau A>48 \pi(g-1)$, then we have the equality

$$
\mathrm{Nul} \phi=\frac{\tau A}{12 \pi}+2-2 g \text {. }
$$

Remark. The main theorem in $[\mathrm{MW}]$ applied to our case says that

$$
\text { Ind } \phi \geq \frac{\tau A}{24 \pi}+g-1 \text {. }
$$

Proof. We associate to each normal field $\xi \in \Gamma(N)$ a section $\psi_{\xi}$ of $\operatorname{End}\left(T \Sigma, T^{\perp} \Sigma\right)$ by the relation

$$
\psi_{\xi}(v)=D_{v} \xi, \quad v \in T \Sigma
$$

It is clear that

$$
\xi \in H^{0}(\Sigma, \mathcal{O}(N)) \text { if and only if } \quad \psi_{\xi}=0 .
$$

Moreover, as we can easily see from (1) that $\psi_{\xi} \circ J=-J \circ \psi_{\xi}, \psi_{\xi}$ defines a section of the line bundle $\kappa^{*} \otimes N$, where $\kappa^{*}$ stands for the conjugate of the canonical line bundle over $\Sigma$. Using the Koszul-Malgrange characterization of the holomorphic structure of this line bundle, we have that $\psi_{\xi}$ will be holomorphic, that is, $\psi_{\xi} \in$ $H^{0}\left(\Sigma, \mathcal{O}\left(\kappa^{*} \otimes N\right)\right)$, if and only if

$$
\left(\nabla \psi_{\xi}\right)(J u, J v)=\left(\nabla \psi_{\xi}\right)(u, v)
$$

for any tangent vectors $u, v \in T \Sigma$. Moreover, from the definition (8) and using (2) and (5), the codifferential of $\psi_{\xi}$ can be computed having that

$$
L \xi-\frac{\tau}{6} \xi=J \delta \psi_{\xi}
$$


Now, if we define a section $T_{\xi}$ of the bundle End $\left(T \Sigma\right.$, End $\left.\left(T \Sigma, T^{\perp} \Sigma\right)\right)$ by

$$
T_{\xi}(u, v)=\left(\nabla \psi_{\xi}\right)(J u, J v)-\left(\nabla \psi_{\xi}\right)(u, v),
$$

then we can state that

$$
\psi_{\xi} \in H^{0}\left(\Sigma, \mathcal{O}\left(\kappa^{*} \otimes N\right)\right) \text { if and only if } \quad T_{\xi}=0 .
$$

From (9) it is easy to conclude that $T_{\xi}(J u, v)=-J T_{\xi}(u, v)$ for any $u, v \in T \Sigma$. In particular, $T_{\xi}$ is a trace zero symmetric tensor. This property of $T_{\xi}$ allows us to identify it with a section of the holomorphic line bundle $\kappa^{2} \otimes N^{*}$.

We are going to compute the codifferential $\delta T_{\xi}$ of the tensor $T_{\xi}$. Using the Ricci identity, the symmetry of $\psi_{\xi}$ and the definition (9), we have

$$
\begin{aligned}
\delta T_{\xi}(v)= & \sum_{i=1}^{2}\left(\nabla T_{\xi}\right)\left(e_{i}, e_{i}, v\right)=\sum_{i=1}^{2}\left(\nabla T_{\xi}\right)\left(e_{i}, v, e_{i}\right) \\
= & \sum_{i=1}^{2}\left\{\left(\nabla^{2} \psi_{\xi}\right)\left(J v, e_{i}, J e_{i}\right)-\left(\nabla^{2} \psi_{\xi}\right)\left(v, e_{i}, e_{i}\right)+R^{\perp}\left(e_{i}, J v\right) \psi_{\xi}\left(J e_{i}\right)\right. \\
& \left.-\psi_{\xi}\left(R\left(e_{i}, J v\right), J e_{i}\right)-R^{\perp}\left(e_{i}, v\right) \psi_{\xi}\left(e_{i}\right)+\psi_{\xi}\left(R\left(e_{i}, v\right) e_{i}\right)\right\} .
\end{aligned}
$$

Now, we use the relation that we had stablished between $T_{\xi}$ and $J$ and the definitions of Gauss and normal curvatures, and obtain

$$
\delta T_{\xi}(v)=-\sum_{i=1}^{2}\left\{J\left(\nabla^{2} \psi_{\xi}\right)\left(J v, e_{i}, e_{i}\right)+\left(\nabla^{2} \psi_{\xi}\right)\left(v, e_{i}, e_{i}\right)\right\}-2\left(K+K^{\perp}\right) \psi_{\xi}(v) .
$$

From (2) and (8) it is straightforward to get

$$
\delta T_{\xi}=-J \psi_{\delta \psi_{\xi}}-\frac{\tau}{6} \psi_{\xi}
$$

So, if we use the expression for $L-\frac{\tau}{6}$ in terms of the codifferential of $\psi_{\xi}$ obtained above, our computation provides us with the following relation:

$$
\delta T_{\xi}=-\psi_{L \xi} .
$$

Now, take the field $\xi$ to be an eigenfield for the Jacobi operator, that is, such that $L \xi+\lambda \xi=0$ for a real number $\lambda$. Hence $\delta T_{\xi}=\lambda \psi_{\xi}$. Using the definition (9), theproperties of $T_{\xi}$ and integration by parts, we obtain

$$
\begin{aligned}
\int_{\Sigma}\left|T_{\xi}\right|^{2} d A & =\int_{\Sigma} \sum_{i, j=1}^{2}\left\langle\left(\nabla \psi_{\xi}\right)\left(J e_{i}, J e_{j}\right)-\left(\nabla \psi_{\xi}\right)\left(e_{i}, e_{j}\right), T_{\xi}\left(e_{i}, e_{j}\right)\right\rangle d A \\
& =-2 \int_{\Sigma} \sum_{i, j=1}^{2}\left\langle\left(\nabla \psi_{\xi}\right)\left(e_{i}, e_{j}\right), T_{\xi}\left(e_{i}, e_{j}\right)\right\rangle d A \\
& =2 \int_{\Sigma} \sum_{j=1}^{2}\left\langle\psi_{\xi}\left(e_{j}\right), \delta T_{\xi}\left(e_{j}\right)\right\rangle d A=2 \lambda \int_{\Sigma}\left|\psi_{\xi}\right|^{2} d A .
\end{aligned}
$$

So, in the case where the eigenvalue $\lambda$ corresponding to the eigenfield $\xi$ is negative, we have shown that $\psi_{\xi}=0$, that is, the field $\xi$ must be a holomorphic section of the line bundle $N$. Then, part b) of Theorem 3 and its proof tell us that $\lambda=-\frac{\tau}{6}$ and

$$
\text { Ind } \phi=2 \operatorname{dim} H^{0}(\Sigma, \mathcal{O}(N))=\frac{\tau A}{12 \pi}+2 g-2
$$


In the case where the field $\xi$ lies on the nullity space $\mathrm{N}(\phi)$, that is, $\lambda=0$, we have from the same integral formula that $T_{\xi}=0$ and, as we remarked before, $\psi_{\xi}$ is a holomorphic section of $\kappa^{*} \otimes N$. In this way, we may define an endomorphism $\psi: \mathrm{N}(\phi) \rightarrow H^{0}\left(\Sigma, \mathcal{O}\left(\kappa^{*} \otimes N\right)\right)$ which maps each $\xi \in \mathrm{N}(\phi)$ to the corresponding $\psi_{\xi}$. Our aim is to see that this map $\psi$ is an isomorphism. In fact, if $\psi(\xi)=0$ for some $\xi \in \mathrm{N}(\phi)$, then from (8), $\xi \in H^{0}(\Sigma, \mathcal{O}(N))$. But, in the proof of Theorem 3, we have shown that all the holomorphic sections of $N$ are eigensections of the Jacobi operator $L$ corresponding to the eigenvalue $-\frac{\tau}{6}$. We conclude that $\xi=0$ and, so, $\psi$ is a monomorphism. Now, take a holomorphic section $\alpha \in H^{0}\left(\Sigma, \mathcal{O}\left(\kappa^{*} \otimes N\right)\right)$, which we will identify with a section of End $\left(T \Sigma, T^{\perp} \Sigma\right)$ satisfying $\alpha \circ J=-J \circ \alpha$. Define the normal field $\xi$ to be $-\frac{6}{\tau} J \delta \alpha$. Using the definition (8), we have

$$
\psi_{\xi}(v)=-\frac{6}{\tau} \sum_{i=1}^{2}\left\{J\left(\nabla^{2} \alpha\right)\left(J v, e_{i}, e_{i}\right)+\left(\nabla^{2} \alpha\right)\left(v, e_{i}, e_{i}\right)\right\}
$$

for any $v \in T \Sigma$. Now, from the Ricci identity, the holomorphicity of $\alpha$ and (2), we obtain

$$
\begin{aligned}
\psi_{\xi}(v)= & \frac{6}{\tau} \sum_{i=1}^{2}\left\{\left(\nabla^{2} \alpha\right)\left(e_{i}, J v, J e_{i}\right)-\left(\nabla^{2} \alpha\right)\left(e_{i}, v, e_{i}\right)+R^{\perp}\left(J v, e_{i}\right) \alpha\left(J e_{i}\right)\right. \\
& \left.-\alpha\left(R\left(J v, e_{i}\right) J e_{i}\right)-R^{\perp}\left(v, e_{i}\right) \alpha\left(e_{i}\right)+\alpha\left(R\left(v, e_{i}\right) e_{i}\right)\right\} \\
= & \frac{12}{\tau}\left(K+K^{\perp}\right) \alpha(v)=\alpha(v) .
\end{aligned}
$$

So, the map $\psi$ is surjective and we get the isomorphism

$$
\mathrm{N}(\phi) \cong H^{0}\left(\Sigma, \mathcal{O}\left(\kappa^{*} \otimes N\right)\right)
$$

Hence the Riemann-Roch theorem and the Serre duality allow us to prove that the nullity of $\phi$ is given by

$$
\operatorname{Nul} \phi=\frac{\tau A}{12 \pi}+2-2 g+2 \operatorname{dim} H^{0}\left(\Sigma, \mathcal{O}\left(\kappa^{2} \otimes N^{*}\right)\right)
$$

The inequality about the nullity follows directly from here. Also, from the formula (3),

$$
c\left(\kappa^{2} \otimes N^{*}\right)=-\frac{\tau A}{24 \pi}+2 g-2
$$

and, so, the holomorphic line bundle $\kappa^{2} \otimes N^{*}$ is negative when $\tau A>48 \pi(g-1)$, and in this case we have the equality.

Corollary 5. Let $\phi: \Sigma \rightarrow M$ be a superminimal immersion from a two-sphere or a torus into a self-dual Einstein four-dimensional manifold with positive scalar curvature $\tau$. Denote by $A$ the area of $\phi$. Then

respectively.

$$
\operatorname{Nul} \phi=\frac{\tau A}{12 \pi}-2 g+2, \quad \text { Ind } \phi=\frac{\tau A}{12 \pi}+2 g-2,
$$

\section{Superminimal immersions into the FOUR-SPHere AND THE COMPLEX PROJECTIVE PLANE}

As we mentioned before, the only compact self-dual Einstein four-dimensional manifolds with positive scalar curvature are the sphere $\mathbb{S}^{4}$ and the complex projective plane $\mathbb{C P}^{2}$. From now on we will consider on $\mathbb{S}^{4}$ the standard metric of 
curvature one and on $\mathbb{C P}^{2}$ the Fubini-Study metric of holomorphic sectional curvature 2. Both these metrics have scalar curvatures $\tau=12$.

We will begin by recalling some important properties of the superminimal surfaces of these two examples. When the ambient space is $\mathbb{S}^{4}$, it is well-known that its twistor space can be identified with the complex projective space $\mathbb{C P}^{3}$ with its complex standard structure, and that the horizontal distribution associated with the twistor projection is given by a complex contact structure (see [ES, G, Sa]). This twistor space has, of course, a big group of complex transformations, and the subgroup consisting of those that preserve the contact distribution is isomorphic to the Lie group $S O(5, \mathbb{C})$. Let $\phi: \Sigma \rightarrow \mathbb{S}^{4}$ be a full superminimal immersion from a compact orientable surface. Then, its twistor lifting $\tilde{\phi}$ can be viewed as a holomorphic immersion from $\Sigma$ into $\mathbb{C P}^{3}$ whose image lies on the contact horizontal distribution. Moreover, as a horizontal complex curve in $\mathbb{C P}^{3}$ which is not linearly full must be a line, which is the twistor lifting of the totally geodesic immersion in $\mathbb{S}^{4}$, we have that $\tilde{\phi}$ is linearly full in $\mathbb{C P}^{3}$. As a consequence, the space of full superminimal immersions into $\mathbb{S}^{4}$ can be constructed by projecting a certain class of linearly full complex curves of the complex projective space $\mathbb{C P}^{3}$. The following properties are well-known:

1) Every minimal immersion of a two-sphere into $\mathbb{S}^{4}$ is superminimal $[\mathrm{Br}, \mathrm{C}]$.

2) The area $A$ of a full superminimal immersion $\phi$ is $4 \pi \operatorname{deg} \tilde{\phi}$. So $A \geq 12 \pi$, and equality holds if and only if $\tilde{\phi}$ is a horizontal rational normal curve in $\mathbb{C P}^{3}[\mathrm{C}, \mathrm{B}, \mathrm{G}]$.

3) The non-compact Lie group $S O(5, \mathbb{C})$ of complex contact transformations of $\mathbb{C P}^{3}$ acts naturally on the space of full superminimal immersions in $\mathbb{S}^{4}$ keeping the area $A$ fixed. This action is transitive on the subspace of lowest area $A=12 \pi$ because any two rational normal curves are always projectively equivalent $[\mathrm{B}, \mathrm{GH}]$. In particular, this subspace is connected. Notice that the space of branched minimal immersions from a two-sphere into $\mathbb{S}^{4}$ with fixed area $4 \pi d$ is connected, as Loo and Kotani have shown $[\mathrm{Lo}, \mathrm{K}]$. But it seems to the authors that their arguments do not work when one considers only unbranched immersions.

4) The Veronese embedding of the real projective plane into the sphere $\mathbb{S}^{4}$ provides a well-known full superminimal immersion from the two-sphere into $\mathbb{S}^{4}$ which has parallel second fundamental form and induced metric with constant Gauss curvature $\frac{1}{3}$. So, this Veronese map is a full superminimal immersion with area $12 \pi$. Hence, one obtains all superminimal immersions into $\mathbb{S}^{4}$ with area $12 \pi$ by deforming it by means of the action of the group $S O(5, \mathbb{C})$. We will refer to this family of superminimal immersions as twistor deformations of the Veronese immersion.

Consider now the case where the ambient space is the complex projective plane $\mathbb{C P}^{2}$. It is also well-known that the twistor space of $\mathbb{C P}^{2}$, as a complex contact manifold, can be identified with the projectified complex tangent bundle $\mathbb{P} T \mathbb{C P}^{2}$, although, of course, the twistor projection $\pi$ is not the natural holomorphic projection $p: \mathbb{P} T \mathbb{C P}^{2} \rightarrow \mathbb{C P}^{2}$. The correspondence which maps each superminimal branched immersion $\phi$ into $\mathbb{C P}^{2}$ to the complex curve of the complex projective plane $\hat{\phi}=p \circ \tilde{\phi}$ is one to one, as Chern and Wolfson, Gauduchon, Eells and Salamon proved [CheW, G, ES]. The complex curve $\hat{\phi}$ is frequently called the directrix curve of $\phi$. The following properties have also been obtained:

1) Every minimal immersion of a two-sphere into $\mathbb{C P}^{2}$ is either a complex immersion or superminimal [CheW].

2) The area $A$ of a full superminimal immersion $\phi$ is $4 \pi \operatorname{deg} \hat{\phi}+2 \pi \operatorname{deg} \phi$. Another useful and non-written expression for the area $A$ can be/ obtained manipulating 
results of Theorem 5.2 of [EGT] together with Plücker formulae: $A=2 \pi \operatorname{deg} \hat{\phi}+$ $2 \pi \operatorname{deg} \hat{\phi}^{*}, \hat{\phi}^{*}$ being the dual curve of $\phi$. Hence, $A \geq 8 \pi$, and equality occurs if and only if $\hat{\phi}$ is a conic [ES, EGT].

3) The non-compact Lie group $P G L(3, \mathbb{C})$ of the complex transformations of $\mathbb{C P}^{2}$ acts, through the directrices curves, on the space of branched full superminimal immersions in $\mathbb{C P}^{2}$ keeping the area $A$ fixed. This action is transitive on the subspace of lowest area $A=8 \pi$ because any two non-degenerate conics in $\mathbb{C P}^{2}$ are projectively equivalent. In particular, this subspace is connected.

4) The totally geodesic totally real embedding of the real projective plane into $\mathbb{C P}^{2}$ provides a full superminimal immersion from the two-sphere into $\mathbb{C P}^{2}$ whose induced metric has constant curvature $\frac{1}{2}$ and hence area $8 \pi$. This immersion will be called the totally real Veronese immersion. So, by deforming this superminimal immersion by the action of the group $P G L(3, \mathbb{C})$, one obtains all superminimal immersions into $\mathbb{C P}^{2}$ with area $8 \pi$. We will refer to this family of superminimal immersions as twistor deformations of the totally real Veronese immersion.

Taking into account Theorem 4, the problem of finding lower bounds for the index and the nullity of a superminimal immersion into the four-sphere or the complex projective plane and characterizing those which attain these bounds is reduced to doing the same for the area. These immersions with lowest index are the natural substitutes for the stable ones, and could represent the equilibrium states for the variational problem that we are considering.

The first results about this subject are due to Simons [Si], who proved that totally geodesic immersions in $\mathbb{S}^{4}$ and totally geodesic complex immersions in $\mathbb{C P}^{2}$ are the minimal immersions with lowest index and nullity (index 2 and nullity 6 for $\mathbb{S}^{4}$ and index 0 and nullity 4 for $\mathbb{C P}^{2}$ ). In some sense, these immersions are degenerate because their images are respectively two dimensional equators in $\mathbb{S}^{4}$ and complex projective lines in $\mathbb{C P}^{2}$. In fact, these are the only superminimal immersions which lie on linear subspaces of these two ambient spaces. So, we will exclude these totally geodesic cases, and hence we will consider full superminimal immersions.

The following result gives a lower bound for the area in terms of the genus of the surface. This is in the spirit of the Brill-Noether theorem and, in fact, the proof consits of an adequate use of this theorem.

Lemma 6. Let $A$ be the area of a conformal full superminimal immersion from a compact Riemann surface of genus $g$ into $\mathbb{S}^{4}$ or $\mathbb{C P}^{2}$. Then, for the generic Riemann surface structure, we have

$$
\frac{A}{4 \pi} \geq\left[\frac{3+3 g}{4}\right]+3
$$

when the ambient space is $\mathbb{S}^{4}$, and

$$
\frac{A}{4 \pi} \geq\left[\frac{2+2 g}{3}\right]+2
$$

when the ambient is $\mathbb{C P}^{2}$.

Proof. If $\phi$ is such an immersion into $\mathbb{S}^{4}$, we have that its twistor lifting $\tilde{\phi}$ is a linearly full horizontal curve in the complex projective space $\mathbb{C P}^{3}$. Hence, because $\operatorname{deg} \tilde{\phi}=\frac{A}{4 \pi}$, using the Brill-Noether theorem (see [GH]) one can obtain the first inequality. On the other hand, if $\phi$ represents an immersion of this type into 
the complex projective plane $\mathbb{C P}^{2}$, we have that its directrix curve $\hat{\phi}$ is a linearly full holomorphic curve in $\mathbb{C P}^{2}$, and we know from the observations above that $\frac{A}{2 \pi}=d+d^{*}$, where $d$ and $d^{*}$ stand for the degree and the class of $\hat{\phi}$ respectively. Using the Brill-Noether theorem again, we finish the lemma.

From Lemma 6, we can see that the inequality about the nullity obtained in Theorem 4 is attained for small genera. In fact, we get $\tau A>48 \pi(g-1)$ when $g \leq 15$ in the case of the sphere and $g \leq 8$ in the case of the complex projective plane. Then, we have

Corollary 7. The nullity of a conformal superminimal immersion from a generic compact Riemann surface of genus $g \leq 15$ into $\mathbb{S}^{4}$ is exactly $\frac{A}{\pi}+2-2 g$.

Corollary 8. The nullity of a conformal superminimal immersion from a generic compact Riemann surface of genus $g \leq 8$ into $\mathbb{C P}^{2}$ is exactly $\frac{A}{\pi}+2-2 g$.

Now, we will be able to characterize those superminimal immersions into $\mathbb{S}^{4}$ and $\mathbb{C P}^{2}$ which attain the lowest index and nullity.

Corollary 9. Let $\phi: \Sigma \rightarrow \mathbb{S}^{4}$ be a superminimal full immersion from a compact orientable surface $\Sigma$ into the four-sphere. Then $\operatorname{Ind} \phi \geq 10$, and equality holds if and only if $\phi$ is the Veronese immersion or one of its twistor deformations.

Corollary 10. If $\phi$ is a full minimal immersion from the two-sphere into $\mathbb{S}^{4}$, then Ind $\phi \geq 10$ and Nul $\phi \geq 14$. Equality holds if and only if $\phi$ is the Veronese immersion or one of its twistor deformations.

The proofs of both corollaries follow directly from Theorem 4 and the properties listed above.

Using a similar reasoning when the ambient space is the complex projective plane, we get the following results.

Corollary 11. Let $\phi: \Sigma \rightarrow \mathbb{C P}^{2}$ be a superminimal full immersion from a compact orientable surface $\Sigma$ into the complex projective plane. Then $\operatorname{Ind} \phi \geq 6$, and equality holds if and only if $\phi$ is the totally real Veronese immersion or one of its twistor deformations.

Corollary 12. If $\phi$ is a full minimal immersion from the two-sphere into $\mathbb{C P}^{2}$, then either $\phi$ is a complex immersion or Ind $\phi \geq 6$ and $\mathrm{Nul} \phi \geq 10$. Equality occurs if and only if $\phi$ is the totally real Veronese immersion or one of its twistor deformations.

Remark. When $\phi$ is the Veronese immersion into either of the ambient spaces $\mathbb{S}^{4}$ or $\mathbb{C P}^{2}$, these results tell us that $\mathrm{Nul} \phi=14$ and $\mathrm{Nul} \phi=10$ respectively. One can easily observe that the infinitesimal isometries of the four-sphere and the complex projective plane contribute to that nullity with seven and five independent fields respectively. So, the moduli space of full minimal immersions with lowest area, up to isometries of the ambient space, has dimension seven in the case of the sphere, and five in the case of the complex projective plane. This is in apparent contradiction with [B], where the dimension computed for this moduli space was ten. It seems to the authors that reparametrizations of the two-sphere were not taken into account. 
When the map $\phi$ is the Veronese immersion we will be able to compute not only the multiplicities of the two first eigenvalues, but the whole of the spectrum of the Jacobi operator. In order to do this, we will prove the following lemma.

Lemma 13. Let $T$ be a trace zero symmetric two-tensor on a Riemannian surface $\Sigma$ of genus zero. Then, there exists an one-form $\alpha$ such that

$$
T(u, v)=(\nabla \alpha)(J u, J v)-(\nabla \alpha)(u, v)
$$

for all $u, v$ tangent to the surface, where $\nabla$ is the connection associated to the metric and $J$ is any complex structure.

Proof. Let $\mathcal{T}_{0} \Sigma$ be the fiber bundle of the trace zero symmetric two-tensors over $\Sigma$. We define a differential operator $D: \Gamma\left(T^{*} \Sigma\right) \rightarrow \Gamma\left(\mathcal{T}_{0} \Sigma\right)$ of order one by the following relation:

$$
(D \alpha)(u, v)=(\nabla \alpha)(J u, J v)-(\nabla \alpha)(u, v)
$$

for any one-form $\alpha$ on $\Sigma$. Notice that this two-tensor $D \alpha$ satisfies $(D \alpha)(J u, J v)$ $=-(D \alpha)(u, v)$, and this is equivalent to being trace zero and symmetric because $\operatorname{dim} \Sigma=2$. Moreover, if $T \in \Gamma\left(\mathcal{T}_{0} \Sigma\right)$, integrating by parts, we have

$$
\begin{gathered}
\int_{\Sigma}\langle D \alpha, T\rangle d A=\int_{\Sigma} \sum_{i, j=1}^{2}\left((\nabla \alpha)\left(J e_{i}, J e_{j}\right)-(\nabla \alpha)\left(e_{i}, e_{j}\right)\right) T\left(e_{i}, e_{j}\right) d A \\
=-2 \int_{\Sigma} \sum_{i, j=1}^{2}(\nabla \alpha)\left(e_{i}, e_{j}\right) T\left(e_{i}, e_{j}\right) d A=2 \int_{\Sigma}\langle\alpha, \delta T\rangle d A .
\end{gathered}
$$

That is, the $L^{2}$-adjoint of the operator $D$ is twice the codifferential $\delta$ acting on $\Gamma\left(\mathcal{T}_{0} \Sigma\right)$. Using Corollary 4.2 in [BE], we have the following orthogonal decomposition:

$$
\Gamma\left(\mathcal{T}_{0} \Sigma\right)=D \Gamma\left(T^{*} \Sigma\right) \oplus \delta^{-1}(0) .
$$

It suffices to see that the fact that $\Sigma$ has genus zero implies that $\delta^{-1}(0)=0$. In fact, if $T \in \Gamma\left(\mathcal{T}_{0} \Sigma\right)$, then $\omega=T\left(\partial_{z}, \partial_{z}\right)(d z)^{2}$ is an order two differential on $\Sigma$, where $z$ is a local isothermal parameter. It is immediate that $\omega$ is holomorphic if and only if $\delta T=0$. We conclude from the Riemann-Roch theorem.

Theorem 14. The eigenvalues $\lambda_{k}$ of the Jacobi operator of the Veronese immersion and their respective multiplicities $m_{k}$ are given by the following sequences:

$$
\lambda_{k}=\frac{1}{3}(k-2)(k+5), \quad m_{k}=2(2 k+3) .
$$

Proof. We start by recalling that the Veronese immersion of the two-sphere $\mathbb{S}^{2}$ into $\mathbb{S}^{4}$ has parallel second fundamental form $\sigma$ and constant length $\frac{2}{\sqrt{3}}$. This implies that the normal vectors $\left\{\frac{1}{\sqrt{3}} \sigma\left(e_{1}, e_{1}\right), \frac{1}{\sqrt{3}} \sigma\left(e_{1}, e_{2}\right)\right\}$ provide us an orthonormal reference, $\left\{e_{1}, e_{2}\right\}$ being a tangent orthonormal reference. We define a linear map $F: \Gamma\left(T^{*} \mathbb{S}^{2}\right) \rightarrow \Gamma\left(T^{\perp} \mathbb{S}^{2}\right)$ given by

$$
F(\alpha)=\sum_{i, j=1}^{2}(\nabla \alpha)\left(e_{i}, e_{j}\right) \sigma\left(e_{i}, e_{j}\right) .
$$

The kernel of $F$ consists of the one-forms $\alpha$ which satisfy

$$
(\nabla \alpha)(J u, J v)=(\nabla \alpha)(u, v) \text { for all } u, v \text { tangent to } \mathbb{S}^{2} .
$$


This means that $\alpha$ is a holomorphic one-form. Now, we want to show that $F$ is a surjective map. In fact, given a normal vector field $\xi$, we have

$$
\xi=\frac{3}{2} \sum_{i, j=1}^{2}\left\langle\sigma\left(e_{i}, e_{j}\right), \xi\right\rangle \sigma\left(e_{i}, e_{j}\right)
$$

But $T(u, v)=\frac{3}{2}\langle\sigma(u, v), \xi\rangle$ is a trace zero symmetric tensor on $\mathbb{S}^{2}$. From Lemma 13 , we have that $T=D \alpha$ for some one-form $\alpha$. So

$$
\begin{aligned}
\xi & =\sum_{i, j=1}^{2}\left\{(\nabla \alpha)\left(J e_{i}, J e_{j}\right)-(\nabla \alpha)\left(e_{i}, e_{j}\right)\right\} \sigma\left(e_{i}, e_{j}\right) \\
& =-2 \sum_{i, j=1}^{2}(\nabla \alpha)\left(e_{i}, e_{j}\right) \sigma\left(e_{i}, e_{j}\right),
\end{aligned}
$$

where we have taken into account part c) of Proposition 1. Hence, $\xi=F(-2 \alpha)$ and $F$ is an epimorphism.

On the other hand, since $\nabla \sigma=0$, for any one-form $\alpha$ on the surface we have

$$
\Delta^{\perp} F(\alpha)=\sum_{i, j, k=1}^{2}\left(\nabla^{3} \alpha\right)\left(e_{k}, e_{k}, e_{i}, e_{j}\right) \sigma\left(e_{i}, e_{j}\right) .
$$

But the Ricci identity gives us

$$
\left(\nabla^{2} \alpha\right)\left(e_{k}, e_{i}, e_{j}\right)=\left(\nabla^{2} \alpha\right)\left(e_{i}, e_{k}, e_{j}\right)-\frac{1}{3} \delta_{i j} \alpha\left(e_{k}\right)+\frac{1}{3} \delta_{k j} \alpha\left(e_{i}\right),
$$

and then we can write

$$
\Delta^{\perp} F(\alpha)=\sum_{i, j, k=1}^{2}\left(\nabla^{3} \alpha\right)\left(e_{k}, e_{i}, e_{k}, e_{j}\right) \sigma\left(e_{i}, e_{j}\right)+\frac{1}{3} F(\alpha) .
$$

Using the Ricci identity again, we obtain

$$
\sum_{k=1}^{2}\left(\nabla^{3} \alpha\right)\left(e_{k}, e_{i}, e_{k}, e_{j}\right)=\sum_{k=1}^{2}\left(\nabla^{3} \alpha\right)\left(e_{i}, e_{k}, e_{k}, e_{j}\right)+\frac{2}{3}(\nabla \alpha)\left(e_{i}, e_{j}\right)-\frac{1}{3} \delta_{i j} \delta \alpha,
$$

where $\delta \alpha$ is the codifferential of the one-form $\alpha$. Putting together these last expressions, we see that

$$
\Delta^{\perp} F(\alpha)=\sum_{i, j, k=1}^{2}\left(\nabla^{3} \alpha\right)\left(e_{i}, e_{k}, e_{k}, e_{j}\right) \sigma\left(e_{i}, e_{j}\right)+F(\alpha) .
$$

But, the Veronese surface has constant Gauss curvature $\frac{1}{3}$. If we represent by $\Delta$ the Laplacian operator acting on one-forms, then

$$
(\Delta \alpha)(v)=\sum_{i=1}^{2}\left(\nabla^{2} \alpha\right)\left(e_{k}, e_{k}, v\right)-\frac{1}{3} \alpha(v)
$$

for any $v$ tangent to the surface. Hence we arrive to the following formula:

$$
\Delta^{\perp} F(\alpha)=F(\Delta \alpha)+\frac{4}{3} F(\alpha)
$$


for any one-form $\alpha$. Now, regarding formula (5) and recalling that in our case

$K=\frac{1}{3}$, we can conclude that the Jacobi operator $L$ of the Veronese immersion satisfies

$$
L \circ F=F \circ(\Delta+4),
$$

where, as we had remarked, $\Delta$ is the Laplacian operator on the one-forms.

The conclusion of the theorem now follows from the knowledge of the spectrum of the Laplacian acting on the one-forms of a two-sphere $\mathbb{S}^{2}$ endowed with a metric of constant Gauss curvature $\frac{1}{3}$ and the fact that, for this metric, the holomorphic one-forms of the sphere are exactly the eigenforms of the Laplacian corresponding to the first eigenvalue $\frac{2}{3}$.

Also when the ambient space is the complex projective plane one can compute the whole of the spectrum of the Jacobi operator for the simplest immersion of this family of lowest area: the totally real Veronese immersion. For this kind of immersions (the totally real ones), $\mathrm{Oh}[\mathrm{O}]$ proved that the Jacobi operator and the Laplacian acting on one-forms are related by

$$
L \circ F=F \circ(\Delta+3),
$$

where $F: \Gamma\left(T^{*} \mathbb{S}^{2}\right) \rightarrow \Gamma\left(T^{\perp} \mathbb{S}^{2}\right)$ is the isomorphism given by

$$
\langle F(\alpha), \xi\rangle=\alpha(\mathcal{I} \xi) \quad \text { for all } \xi \in \Gamma\left(T^{\perp} \mathbb{S}^{2}\right),
$$

$\mathcal{I}$ being the complex structure on $\mathbb{C P}^{2}$ which, in this totally real case, defines an isomorphism between the normal and the tangent bundles on the surface. Then, since our surface has constant curvature $\frac{1}{2}$, we have:

Remark. The eigenvalues $\lambda_{k}$ of the Jacobi operator of the totally real totally geodesic immersion from $\mathbb{S}^{2}$ into $\mathbb{C P}^{2}$ and their respective multiplicities $m_{k}$ are given by

$$
\lambda_{k}=\frac{1}{2}(k-2)(k+3), \quad m_{k}=2(2 k+1) .
$$

\section{REFERENCES}

[AHS] M.F. Atiyah, N.J. Hitchin, I.M. Singer, Self-duality in four-dimensional Riemannian geometry, Proc. Roy. Soc. London Ser. A, 362(1978), 425-461. MR 80d:53023

[B] J.L.M. Barbosa, On minimal immersions of $\mathbb{S}^{2}$ into $\mathbb{S}^{2 m}$, Trans. Amer. Math. Soc., 210(1975), 75-106. MR 51:11362

[Be1] A.L. Besse, Géométrie Riemannienne en Dimension 4, Cedic-Fernand Nathan, 1981, Paris. MR 86i: 53020

[Be2] A.L. Besse, Einstein Manifolds, Springer-Verlag, 1987, Berlin. MR 88f:53087

[Br] R.L. Bryant, Conformal and minimal immersions of compact surfaces into the 4-sphere, J. Differential Geometry, 17(1982), 455-473. MR 84a:53062

[BE] M. Berger, D. Ebin, Some decompositions of the space of symmetric tensors on a Riemannian manifold, J. Differential Geometry, 3(1969), 379-392. MR 42:993

[C] E. Calabi, Minimal immersions of surfaces in Euclidean spheres, J. Differential Geometry, 1(1967), 111-125. MR 38:1616

[Ch] I. Chavel, Eigenvalues in Riemannian Geometry, Academic Press, 1984, Orlando. MR 86g:58140

[CheW] S.S. Chern, J.G. Wolfson, Minimal surfaces by moving frames, Amer. J. Math., 105(1983), 59-83. MR 84i:53056

[E] N. Ejiri, The index of minimal immersions of $\mathbb{S}^{2}$ into $\mathbb{S}^{2 n}$, Math. Z., 184(1983), 127-132. MR 85b:53062

[ES] J. Eells, S. Salamon, Twistorial constructions of harmonic maps of surfaces into fourmanifolds, Ann. Scuola Norm. Sup. Pisa, 12(1985), 589-640. MR 87i:58042 
[EGT] J.H. Eschenburg, I.V. Guadalupe, R.A. Tribuzy, The fundamental equations of minimal surfaces in $\mathbb{C P}^{2}$, Math. Ann., 270(1985), 571-598. MR 86m:58041

[FC] D. Fischer-Colbrie, On complete minimal surfaces with finite Morse index in threemanifolds, Invent. Math., 82(1985), 121-132. MR 87b:53090

[FCS] D. Fischer-Colbrie, R. Schoen, The structure of complete stable minimal surfaces in 3-manifolds of non-negative scalar curvature, Comm. Pure Appl. Math., 33(1980), 199211. MR 81i:53044

[Fr] T. Friedrich, On surfaces in four-spaces, Ann. Global Anal. Geom., 2(1984), 257-287. MR 86h:53061

[FrG] T. Friedrich, R. Grünewald, On Einstein metrics on the twistor space of a fourdimensional Riemannian manifold, Math. Nachr., 123(1985), 55-60. MR 87c:53093

[G] P. Gauduchon, Les immersions super-minimales d'une surface compacte dans une variété riemannienne orientée de dimension 4, Astérisque, 154-155(1987), 151-180. MR 89g:58052

[GH] P. Griffiths, J. Harris, Principles of Algebraic Geometry, Wiley, 1978, New York. MR 80b: 14001

[H] N.J. Hitchin, Kählerian twistor spaces, Proc. Lond. Math. Soc., 43(1981), 133-150. MR 84b:32014

[K] M. Kotani, Connectedness of the space of minimal 2-spheres in $\mathbb{S}^{n}(1)$., Proc. Amer. Math. Soc., 120(1994), 803-810. MR 94e:58033

[KM] J.L. Koszul, B. Malgrange, Sur certaines structures fibrées complexes, Arch. Math., 9(1958), 102-109. MR 24:A1729

[L] B. Lawson, Lectures on Minimal Submanifolds, Publish or Perish Inc., 1976, Berkeley. MR 82d:53035

[Lo] B. Loo, The space of harmonic maps of $\mathbb{S}^{2}$ into $\mathbb{S}^{4}$, Trans. Amer. Math. Soc., 313(1989), 81-102. MR 90k:58050

[MM] M.J. Micallef, J.D. Moore, Minimal two-spheres and the topology of manifolds with positive curvature on totally isotropic twp-planes, Ann. of Math., 127(1988), 199-227. MR 89e:53088

[MW] M.J. Micallef, J.G. Wolfson, The second variation of area of minimal surfaces in fourmanifolds, Math. Ann., 295(1993), 245-267. MR 94c:58035

$[\mathrm{MoR}]$ S. Montiel, A. Ros, Schrödinger operators associated to a holomorphic map, Lecture Notes in Mathematics, 1481(1991), 147-174. MR 93k:58053

[O] Y.G. Oh, Second variation and stabilities of minimal Lagrangian submanifolds in Kähler manifolds, Invent. Math., 101(1990), 501-519. MR 91f:58022

[SY] R. Schoen, S.T. Yau, Existence of incompressible minimal surfaces and the topology of three dimensional manifolds with non-negative scalar curvature, Ann. of Math., 110(1979), 127-142. MR 81k:58029

[Sa] S. Salamon, Topics in four-dimensional Riemannian geometry, Lectures Notes in Mathematics, 1022(1982), 34-124. MR 85j:53002

[Si] J. Simons, Minimal varieties in Riemaniann manifolds, Ann. of Math., 88(1968), 62-105.

[SiuY] Y.T. Siu, S.T. Yau, Compact Kähler manifolds of positive bisectional curvature, Invent. Math., 59(1980), 189-204. MR 81h:58029

[U] F. Urbano, Minimal surfaces with low index in the three-dimensional sphere, Proc. Amer. Math. Soc., 108(1990), 989-992. MR 90h:53073

Departamento de Geometría y Topología, Universidad de Granada, E-18071 Granada, SPAIN

E-mail address: smontiel@goliat.ugr.es

Departamento de Geometría y Topología, Universidad de Granada, E-18071 Granada, SPAIN

E-mail address: furbano@goliat.ugr.es 